\title{
Ultrasound assessment of the suspected scaphoid fracture
}

\author{
D. J. DACRUZ, R. H. TAYLOR, B. SAVAGE \& G. G. BODIWALA \\ Department of Accident and Emergency, Leicester Royal Infirmary, Leicester, England
}

\section{SUMMARY}

A prospective study of 111 patients thought to have sustained a recent scaphoid fracture on clinical grounds but who were radiologically negative was undertaken over a period of 7 months. All such patients were subjected to ultrasound scanning within a week of their injury under double blind conditions. All patients were re-X-rayed 2-3 weeks after their injury. The authors' results suggest that ultrasonic diagnosis of the possibly fractured scaphoid is unreliable.

\section{INTRODUCTION}

Ultrasonic energy is obtained by applying an oscillating electrical voltage to a crystal. This crystal will have been ground to precise dimensions so that it expands and contracts at the same frequency as the applied electrical voltage. This phenomenon, known as the piezo-electric effect, is thought to be capable of causing periosteal pain, either by generating heat at the soft-tissue/bone interface (Oakley, 1978) or by causing vibration at the fracture site.

Though its mode of action is unclear, ultrasonic energy in the therapeutic range is known to be capable of producing pain or severe tingling when applied to fractures less than 2 weeks old (Nitz \& Scoville, 1980; Bedford et al., 1982; Shenouda \& England, 1987). In the latter two studies, a strong but not absolute correlation was found between the presence of a recent scaphoid fracture and pain.

A simple, cost-effective method of identifying a fractured scaphoid when it is not radiologically evident has long been sought in order to avoid unnecessarily immobilizing patients wrists in plaster on clinical grounds alone. It was decided to apply ultrasonic energy, in the therapeutic range, to all possible scaphoid fractures in the accident and emergency department, assess results and compare findings with previously published data.

Correspondence: $\mathrm{Mr}$ D. F. DaCruz, Department of Accident and Emergency, Leicester Royal Infirmary, LE1 $5 W W$, England 
In order to first accustom ourselves with the technique, a number of patients with known scaphoid fractures were subjected to ultrasonic energy prior to commencing this study. It was found possible to cause pain/tingling in a large number of these patients.

\section{PATIENTS AND METHODS}

All patients who were suspected to have sustained a scaphoid fracture on clinical grounds (post traumatic pain, tenderness and swelling in the anatomical snuffbox) were studied. Five views of the scaphoid were taken: an Antero-posterior, Lateral, Obliques in $15^{\circ}$ of pronation and supination, (Kessler et al., 1960) and an oblique view with radial deviation. Patients who had definite fractures on radiography were excluded from the study and referred for appropriate treatment.

Only patients with normal radiographs or non-diagnostic lines in the scaphoid were subjected to further assessment using ultrasonic energy, always within a week of the injury. While awaiting the ultrasonic test, the wrist was rested in a sling or removable splint. All patients were assessed by the first author prior to the test. Neither the patient nor the Physiotherapist was aware of clinical or radiological findings at the time of ultrasonic assessment.

Ultransoic diagnosis was carried out by a single senior physiotherapist using a Therasonic machine (Therasonic Double-5, Elctro-Medical Supplies UK). In keep-o ing with the experience of Bedford et al. (1982), ultrasonic energy at $0.5-1.5 \mathrm{~W} / \mathrm{cm}^{2}$ anş a frequency of $1 \mathrm{MHz}$ was applied to the skin overlying the anatomical snuffbox. water-based coupling medium was employed and the probe used had a diameter of $0.75 \mathrm{~cm}$. Patients were asked to compare the sensation produced with that obtained on the same area of the opposite, un-injured limb. The test was deemed positive when pain or severe tingling was experienced on the injured side alone.

For purposes of this study, patients with persistent signs in their wrists indicative of a scaphoid fracture had their wrists immobilized in a scaphoid plaster regardless of the ultrasonic assessment. All patients had repeat radiographs obtained 2-3 weeks after their injury regardless of radiological or ultrasonic findings. Radiographs were reported on by a consultant radiologist sequentially at the end of the study.

\section{RESULTS}

A total of 111 patients were studied, 54 of whom were men. The average age was 30 (range: 10-72 years).

On reviewing radiographs just prior to application of ultrasound, it became apparent that a total of nine fractures had been missed by the casualty officers at the patients first visit (Table 1). They comprised three scaphoid fractures, one radial styloid fracture, three distal intra-articular fractures of the radius and two triquetral fractures. These patients were not excluded from the study as they were precisely the group of patients in whom it was hoped that ultrasonic testing would produce strong evidence of a fracture. 
Table 1 Results: ultrasound assessment of fractures

\begin{tabular}{lccc}
\hline Fracture & $\begin{array}{c}\text { Missed by S.H.O.* } \\
\text { Seen by S.R.** }\end{array}$ & $\begin{array}{c}\text { Total no. of fractures } \\
\text { apparent at 3 weeks }\end{array}$ & $\begin{array}{c}\text { Ultrasound } \\
\text { positive }\end{array}$ \\
\hline Scaphoid & 3 & 6 & 2 \\
Radial styloid & 1 & 2 & 0 \\
Distal radius & 3 & 3 & 0 \\
Trapezium & 0 & 1 & 0 \\
Triqetral & 2 & 3 & 1 \\
\hline
\end{tabular}

*Senior house officer.

**Senior registrar.

Repeat radiographs of patients' wrists taken $2-3$ weeks after their injury revealed a total of 15 fractures, nine of which had been previously identified. The six fractures that were inapparent on initial radiographs were two possible distal pole fractures (both in children), one scaphoid-waist fracture, one radial styloid fracture and one triquetral flake fracture (Table 1). Thus, six of the 15 fractures involved the scaphoid bone but only three of these were apparent to the authors when they reviewed patients just prior to the ultrasonic test.

Only two scaphoid fractures and one triquetral fracture were positive on ultrasonic testing. For suspected scaphoid fractures, therefore, sensitivity of ultrasonic testing was $33 \%$ (four false negatives out of six fractures). A total of 41 patients were positive on ultrasonic testing and 38 of these had no evidence of a fracture.

It was possible to make specific soft tissue diagnoses in 32 patients (Table 2). Ultrasonic testing was found to be neither specific nor sensitive for any of these pathologies.

\section{DISCUSSION}

The need for a test that is simple, cost-effective and preferably free of any radiational problems has long been recognized in the positive diagnosis of a clinically suspected

Table 2 Results: ultrasound assessment of specific soft tissue injuries

\begin{tabular}{|c|c|c|}
\hline Injury & $\begin{array}{l}\text { Ultrasound } \\
\text { positive }\end{array}$ & $\begin{array}{c}\text { Ultrasound } \\
\text { negative }\end{array}$ \\
\hline Extensor pollicis longus tenosynovitis & 1 & 4 \\
\hline Abductor pollicis longus tenosynovitis & 5 & 5 \\
\hline Extensor communis tenosynovitis & 0 & 4 \\
\hline Felxor pollicis longus tenosynovitis & 3 & 3 \\
\hline Sprain metacarpophalangeal joint of thumb & 2 & 2 \\
\hline Traumatic ganglions & 1 & 2 \\
\hline
\end{tabular}


scaphoid fracture. Ultrasound certainly is inexpensive, easy to apply and free of side effects, but it cannot be deemed specific or sensitive for undisplaced, hairline scaphoid fractures. Indeed, there were four false negatives in this group and, in the authors' opinion, this does render the test valueless. Both ultrasonically positive scaphoid fractures were obvious on initial radiographs. Shenouda \& England (1987) disagree with some of our findings. They were able to identify 12 patients who had their scaphoid fractures diagnosed using ultrasound before radiographic evidence was apparent. The amount of ultrasonic energy they employed was twice that used in our study and they studied a group of patients the majority of whom had scaphoid fractures apparent on their initial radiographs. There seems to be little point in subjecting patients with radiologically apparent scaphoid fractures to ultrasonic energy from the $\vec{a}$ point of view of diagnosis. Ultrasonic energy in the context of diagnosing scaphoid fractures is not an imaging technique and, therefore, can never replace radiology: knowledge of the morphology of the fracture is all important. Importantly, in their study, four patients had clear fractures visible on their radiographs but were negative ultrasonically.

Bedford et al. (1982) had a false positive rate of $43.6 \%$ and only one patient had a negative test in the presence of a radiologically confirmed recent fracture. The authors have been unable to reproduce these results. Ultrasonic evaluation of a recent, clinically suspected scaphoid fracture is not a
reliable test.

\section{REFERENCES}

Bedford A. F., Glasgow M. M. \& Wilson J. N. (1982) Ultrasonic assessment of fractures and its use in the diagnosis of a suspected scaphoid fracture. Injury 14, 180-2.

Kessler I., Silberman Z., Heller J. et al. (1960) Diagnostic considerations in fractures of the carpal scaphoid bone. Surgery, Gynaecology and Obstetrics 110, 117-20.

Nitz A. J. \& Scoville C. R. (1980) Use of ultrasound in early detection of stress fractures of the medial tibial plateau. Military Medicine 145, 844-6.

Oakley E. M. (1978) Dangers and contra-indications of therapeutic ultrasound. Physiotherapy 64, 173-9.

Shenouda N. A. \& England J. P. S. (1987) Ultrasound in the diagnosis of scaphoid fractures. fournal of Hand Surgery 12B, 43-5. 